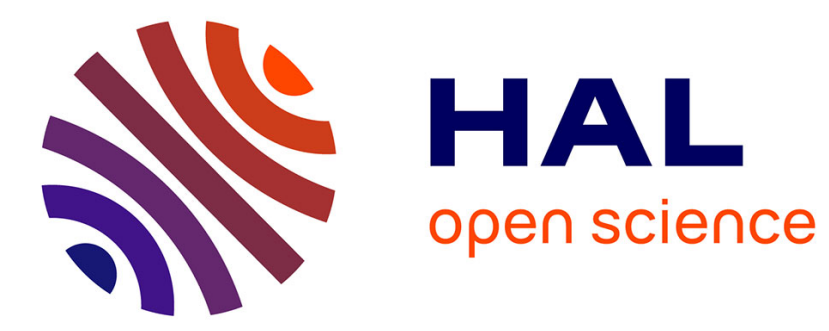

\title{
Syndrome de la maladie post-orgasmique
}

Blaise Bignami, T. Honoré, N. Turmel, R. Haddad, L. Weglinski, Gérard Amarenco, Frédérique Le Breton

\section{To cite this version:}

Blaise Bignami, T. Honoré, N. Turmel, R. Haddad, L. Weglinski, et al.. Syndrome de la maladie post-orgasmique. Progrès en Urologie, 2017, 27 (7), pp.446-448. 10.1016/j.purol.2017.03.007 . hal01960819

\section{HAL Id: hal-01960819 https://hal.sorbonne-universite.fr/hal-01960819}

Submitted on 19 Dec 2018

HAL is a multi-disciplinary open access archive for the deposit and dissemination of scientific research documents, whether they are published or not. The documents may come from teaching and research institutions in France or abroad, or from public or private research centers.
L'archive ouverte pluridisciplinaire $\mathbf{H A L}$, est destinée au dépôt et à la diffusion de documents scientifiques de niveau recherche, publiés ou non, émanant des établissements d'enseignement et de recherche français ou étrangers, des laboratoires publics ou privés. 


\section{Postorgasmic Illness Syndrome : revue de la littérature}

Postorgasmic Illness Syndrome: Literature Review

Blaise BIGNAMI ${ }^{a}$, Frédérique LE BRETON ${ }^{\mathrm{a}}$, Gérard AMARENCO ${ }^{\mathrm{a}}$

Sorbonne Universités, UPMC Univ Paris 06, AP-HP, GRC 01, Groupe de Recherche Clinique en

Neuro-Urologie (GREEN), Hôpital Tenon, Service de Neuro-Urologie, 4 Rue de la Chine, 75020 Paris,

France

\section{Auteur:}

Blaise Bignami

Service de Neuro-Urologie

AP-HP, Hôpital Universitaire Tenon

4, Rue de la Chine

75020 Paris, France

Phone: +33-1-56-01-70-40

Fax : $+33-1-56-01-72-30$

E-mail: blaise.bignami@aphp.fr 
RESUME :

Le "postorgasmic illness syndrome" (POIS) ou "syndrome de la maladie post-orgasmique " (SMPO) est un syndrome rare décrit récemment qui regroupe un ensemble de symptômes cliniques physiques ou cognitifs - apparaissant électivement après une éjaculation ou un orgasme et pouvant perdurer plusieurs jours. Les étiologies et les mécanismes physiopathologiques du POIS restent discutés. Une allergie à une des protéines composant le liquide séminal ou à une protéine excrétée pendant l'éjaculation, une origine psychosomatique, un équivalent migraineux ou épiletique, et une dysrégulation végétative restent les mécanismes les plus évoqués.

Mots-clés : Postorgasmic Illness Syndrome

Postorgasmic illness syndrome (POIS) is a rare and recently described syndrome. It gathers a set of clinical symptoms - physical or cognitive - that occur electively after an ejaculation or an orgasm and may last several days. The etiologies and physiopathological mechanisms of POIS remain discussed. An allergy to one of the proteins composing the seminal fluid or to a protein excreted during ejaculation, a psychosomatic origin, a migrainous or epileptic equivalent, and a vegetative dysregulation remain the most evoked mechanisms.

Key words : Postorgasmic Illness Syndrome 


\section{Postorgasmic Illness Syndrome}

Le POIS a été rapporté et décrit pour la première fois par Waldinger et Schweitzer en $2002^{1}$. On en recense aujourd'hui 55 cas comprenant les deux cas initiaux rapportés par Waldinger et Schweitzer ${ }^{1}$, deux cas rapportés par Ashby et Goldmeier ${ }^{2}$ en 2010, un cas rapporté par Attia et al ${ }^{3}$, un cas rapporté par Puerta Suarez et Cardona Maya en $2013^{4}$, un cas rapporté par Jiang et al en $2015^{5}, 45$ cas récemment rapportés par Waldinger ${ }^{9}$ et 3 cas rapportés par nous-même ${ }^{13}$.

\section{Critères diagnostiques :}

Récemment, Waldinger ${ }^{6}$ a proposé 5 critères pouvant faire évoquer ce syndrome. Premièrement, il faut que le patient présente au moins un des symptômes parmi : une sensation d'état grippal ou d'extrême fatigue, une faiblesse musculaire, un état fébrile ou des frissons, des troubles de I'humeur, une irritabilité, des troubles de la mémoire, des difficultés de concentration, un discours incohérent, un prurit nasal et/ou oculaire. Deuxièmement, l'ensemble de ces symptômes doit apparaître immédiatement (dans la seconde), peu de temps après (quelques minutes) ou dans les heures suivant une éjaculation initiée par un coït, et/ou une masturbation, et/ou spontanément (durant le sommeil par exemple). Troisièmement, ces symptômes doivent toujours ou presque toujours (dans plus de 90\% des cas) survenir après une éjaculation. Quatrièmement, la majorité de ces symptômes doit durer entre 2 et 7 jours. Cinquièmement, les symptômes doivent disparaître spontanément.

\section{Etiopathogénie :}

L'étiopathogénie du POIS n'est pas encore clairement définie. Une théorie tend à rapporter les symptômes à une réaction allergique ${ }^{1}$. En effet, selon Waldinger et Schweitzer, une réaction allergique consécutive à la libération de substances spécifiques dans le sang après une éjaculation et un orgasme pourrait se produire et causer les symptômes du POIS. D'autres théories ont été proposées comme un désordre d'origine psychosomatique, des troubles hormonaux avec un déficit en cortisol ou testostérone, une hypothyroïdie, une hyperprolactinémie, des troubles du système sérotoninergique ou adrénergique (Dean et Ashworth), un équivalent migraineux ou épileptique ou encore une dysautonomie ${ }^{13}$. Il est en effet probable que ces symptômes puissent s'intégrer dans le cadre d'une dysrégulation passagère du système nerveux autonome car il est bien connu que l'éjaculation déclenche un véritable " orage végétatif " avec au décours de l'orgasme une augmentation de l'activité sympathique avec libération de noradrénaline ${ }^{7}$ dans le 
liquide céphalo-rachidien et le plasma. II est probable que d'autres catécholamines et neurotransmetteurs soient également impliqués dans l'excitation et libérés après l'orgasme. II a également été démontré que plusieurs neurotransmetteurs comme la dopamine, la noradrénaline, les opioïdes et la sérotonine jouaient un rôle tantôt stimulateur tantôt inhibiteur sur l'activité sexuelle ${ }^{8}$. Leurs éventuelles contributions dans le POIS sont à ce jour encore inconnues.

Les autres hypothèses qui peuvent être envisagées sont donc neurologiques avec tout d'abord une comitialité partielle déclenchée par l'éjaculation et d'autre part une " migraine sans migraine ". En effet, les symptômes cliniques décrits sont compatibles avec un état post-critique épileptique. II est de plus bien connu qu'il existe de nombreuses circonstances déclenchantes des épisodes migraineux : migraine de week-end, migraine coïtale, migraine cataméniale, migraine de repos, migraine de stress, migraine d'altitude, migraine par privation de café, migraine alcooldépendante, etc... Certains phénomènes migraineux sont même électivement induits par des stimuli spécifiques : aura visuelles induites par une stimulation lumineuse brutale tel un flash ou un rayon de soleil. Le déclenchement d'une crise migraineuse complexe chez certains sujets prédisposés par une éjaculation est ainsi concevable.

Les origines allergiques ou encore immunologiques sont également discutées. Dans une étude de Waldinger ${ }^{9}$ regroupant 45 patients suspects de POIS, 58\% des patients avaient d'autres allergies mais la moyenne des IGE intra-sériques des patients non-atopiques était normale.

L'hypothèse d'une défaillance du système immunologique, dont l'implication dans l'activité sexuelle semble démontrée avec une activation du système immunologique lors de l'orgasme par libération de certains cellules (lymphocytes B et $\mathrm{T}$ ) dans le sang ${ }^{10}$, est également discutée dans cette même étude puisque $88 \%$ des patients présentaient un prick-test positif à leur propre sperme $^{9}$. Cette hypothèse immuno-allergique est corroborée par le cas $^{11} \mathrm{~d}^{\prime}$ une femme ayant déclenché des réactions allergiques après exposition au sperme de son partenaire ainsi que par l'efficacité partielle d'une désensibilisation au sperme chez cette même femme ${ }^{11}$ ainsi que chez deux patients ${ }^{12}$.

\section{Thérapeutique :}

Sur le plan thérapeutique, outre la désensibilisation en cas d'allergie au sperme ${ }^{12,6}$, certaines médications peuvent être essayées comme les anti-inflammatoires non-stéroïdiens, le tramadol, les inhibiteurs sélectifs de la recapture de la sérotonine ${ }^{1,2}$. Il peut aussi être envisagé d'essayer 
des traitements neuroleptiques, antihistaminiques, anticholinergiques et anticholinestérasiques ${ }^{13}$ voire l'introduction d'un traitement anti-migraineux ou anti-épileptique d'épreuve.

\section{CONCLUSION}

Le POIS est une maladie rare et probablement sous-diagnostiquée car encore méconnue.

Au-delà des mécanismes physiopathologiques classiquement suspectés (auto-allergie au liquide séminal, dysfonctionnement psychique), on a pu récemment évoquer des hypothèses spécifiquement neurogéniques telles une dysautonomie spécifique, des crises comitiales partielles avec état post-critique et des crises de migraine complexe. Ces hypothèses permettent d'imaginer des traitements d'épreuve spécifiques.

\section{CONFLITS D'INTERETS :}

Nous ne rapportons aucun conflit d'intérêts. 


\section{REFERENCES}

1. Waldinger, M. D., \& Schweitzer, D. H. (2002). Postorgasmic illness syndrome: Two cases. Journal of Sex \&Marital Therapy, 28(3), 251-255.

2. Ashby, J., \& Goldmeier, D. (2010). CASE REPORT: Postorgasm Illness Syndrome-A Spectrum of Illnesses. The journal of sexual medicine, 7(5), 1976-1981.

3. Attia, A. M., Yasien, H. A., \& Al-Ziny, M. H. (2013). Post-orgasmic illness syndrome: a case report. F1000Research, 2.

4. Puerta, S. J., \& Cardona, M. W. (2013). Postorgasmic illness syndrome: semen allergy in men. Actas urologicas espanolas, 37(9), 593-593.

5. Jiang, N., Xi, G., Li, H., \& Yin, J. (2015). Postorgasmic Illness Syndrome (POIS) in a Chinese Man: No Proof for IgE-Mediated Allergy to Semen. The journal of sexual medicine, 12(3), 840-845.

6. Waldinger, M. D. (2016). Post orgasmic illness syndrome (POIS). Translational Andrology and Urology, 5(4), 602-606.

7. Kruger T, Schiffer B, Eikermann M, Haake P, Gizewski E, Schedlowski M. Serial neurochemical measurement of cerebrospinal fluid during the human sexual response cycle. Eur J Neurosci 2006; 24:3445-52.

8. Pfaus, J. G. (2009). Reviews: Pathways of sexual desire. The journal of sexual medicine, 6(6), 1506-1533.

9. Waldinger, M. D., Meinardi, M. M., Zwinderman, A. H., \& Schweitzer, D. H. (2011). Postorgasmic Illness Syndrome (POIS) in 45 Dutch caucasian males: clinical characteristics and evidence for an immunogenic pathogenesis (Part 1).The journal of sexual medicine, 8(4), 1164-1170.

10. Haake, P., Krueger, T. H., Goebel, M. U., Heberling, K. M., Hartmann, U., \& Schedlowski, M. (2004). Effects of sexual arousal on lymphocyte subset circulation and cytokine production in man. Neuroimmunomodulation, 11(5), 293-298.

11. Lee, J., Kim, S., Kim, M., Chung, Y. B., Huh, J. S., Park \& Kim, J. H. (2008). Anaphylaxis to husband's seminal plasma and treatment by local desensitization. Clinical and Molecular Allergy, 6(1), 1.

12. Waldinger, M. D., Meinardi, M. M., \& Schweitzer, D. H. (2011). Hyposensitization therapy with autologous semen in two Dutch caucasian males: beneficial effects in Postorgasmic Illness Syndrome (POIS; Part 2).The journal of sexual medicine, 8(4), 1171-1176.

13. Bignami B, Honore T, Turmel N, Haddad R, Weglinski L, Le Breton F, Amarenco G. Postorgasmic illness syndrome. Prog Urol. 2017 Jun;27(7):446-448. 